\title{
QCD coupling: scheme variations and tau decays
}

\section{Ramon Miravitllas*}

IFAE, BIST, Campus UAB, 08193 Bellaterra (Barcelona), Catalonia (Spain)

E-mail: rmiravitllaseifae.es

\section{Matthias Jamin}

ICREA, Pg. Lluís Companys 23, 08010 Barcelona, Catalonia (Spain)

IFAE, BIST, Campus UAB, 08193 Bellaterra (Barcelona), Catalonia (Spain)

E-mail: jamineifae.es

We introduce a QCD coupling redefinition which has a simple scheme transformation. As an application, we discuss possible improvements on perturbative predictions of QCD physical quantities. In particular, we focus our attention to the Adler function, which is relevant for the extraction of $\alpha_{\mathrm{s}}$ from tau decays.

$\alpha_{s}$ (2019): Workshop on precision measurements of the QCD coupling constant 11-15 February, 2019

Trento, Italy

\footnotetext{
* Speaker.
} 


\section{The $C$-scheme coupling}

We consider the Adler function [1], which governs theoretical predictions of the inclusive decay rate of tau leptons into hadronic final states [2]. After proper normalisation, a perturbative expansion for the Adler function is given by $D(a)=1+a+\mathscr{O}\left(a^{2}\right)$, where $a=\alpha_{\mathrm{s}}\left(Q^{2}\right) / \pi$ and $Q$ is the momentum transfer of the related physical process.

In the large- $\beta_{0}$ approximation (see [3] for a review), this function has the following Borel integral representation $[4,5]:{ }^{1}$

$$
D(a)=\frac{2}{\beta_{1}} \int_{0}^{\infty} \mathrm{d} u e^{-2 u /\left(\beta_{1} a\right)} B[D](u),
$$

where

$$
B[D](u)=\frac{32}{3} \frac{e^{-C u}}{2-u} \sum_{k \geq 0} \frac{(-1)^{k} k}{\left[k^{2}-(1-u)^{2}\right]^{2}} .
$$

$C$ parametrises the scheme dependence of $a$, arising from the renormalisation of the gluon chain $1 /\left(1+\Pi_{0}\right)$. A constant $C$ remains after subtracting the divergence from the fermion loop in dimensional regularisation:

$$
\Pi_{0}\left(k^{2}\right)=-\beta_{1} \alpha_{\mathrm{s}}\left[\log \left(-\frac{k^{2}}{\mu^{2}}\right)+C\right] .
$$

In the $\overline{\mathrm{MS}}$ scheme, $C=-5 / 3$.

Because the Adler function is a physical quantity, it is independent of theoretical conventions. In particular, the Borel integral in Eq. (1) is independent of $C$ and we conclude that the combination $2 /\left(\beta_{1} a\right)+C$ has to be $C$ invariant. Therefore, the $C$ dependence of the coupling $a$ is given by

$$
\frac{1}{a(C)}=\frac{1}{a(C=0)}-\frac{\beta_{1}}{2} C
$$

Our goal is to define a new coupling in full QCD with similar scheme properties to those in Eq. (4). For that, we define the scale invariant $\Lambda_{\mathrm{QCD}}$ parameter

$$
\Lambda_{\mathrm{QCD}}=Q e^{-1 /\left(\beta_{1} a\right)} a^{-\beta_{2} / \beta_{1}^{2}} \exp \left(\int_{0}^{a} \frac{\mathrm{d} a}{\tilde{\beta}(a)}\right)
$$

where

$$
\frac{1}{\tilde{\beta}(a)}=\frac{1}{\beta(a)}-\frac{1}{\beta_{1} a^{2}}+\frac{\beta_{2}}{\beta_{1}^{2} a}
$$

is free of singularities at $a=0$.

Although $\Lambda_{\mathrm{QCD}}$ by definition is scale independent, it does depend on the scheme. If the coupling is $a$ in one scheme and $a^{\prime}$ in another so that both couplings are related by $a^{\prime}=a+c_{1} a^{2}+$ $\mathscr{O}\left(a^{3}\right)$, then the scheme transformation of $\Lambda_{\mathrm{QCD}}$ is given by [6]

$$
\Lambda^{\prime}=\Lambda e^{c_{1} / \beta_{1}}
$$

where $\Lambda\left(\Lambda^{\prime}\right)$ is the $\Lambda_{\mathrm{QCD}}$ parameter in the $a\left(a^{\prime}\right)$ coupling. The $\Lambda_{\mathrm{QCD}}$ parameter only depends on $c_{1}$ and is insensitive to the rest of the expansion coefficients.

\footnotetext{
${ }^{1}$ In our notation, the $\beta$ coefficients are defined through $\beta(a)=-\mu \mathrm{d} a / \mathrm{d} \mu=\beta_{1} a^{2}+\beta_{2} a^{3}+\ldots$
} 
We fix the coupling $a$ in a specific scheme and define a new coupling $\hat{a}$ through

$$
f(\hat{a})=\beta_{1} \log \left(\frac{Q}{\Lambda_{\mathrm{QCD}}}\right)+\frac{\beta_{1}}{2} C,
$$

where $f$ is some function to be specified later. The right hand side of the equation has a very simple scheme transformation originating from Eq. (7), which for convenience we reparametrised in terms of $C$ instead of $c_{1}$. Thus, the new coupling $\hat{a}$ has the same property regardless of the choice of $f$.

Combining Eq. (5) and Eq. (8), we find

$$
f(\hat{a})-\frac{\beta_{1}}{2} C=\beta_{1} \log \left(\frac{Q}{\Lambda_{\mathrm{QCD}}}\right)=\frac{1}{a}+\frac{\beta_{2}}{\beta_{1}} \log (a)-\beta_{1} \int_{0}^{a} \frac{\mathrm{d} a}{\tilde{\beta}(a)},
$$

so we choose

$$
f(\hat{a})=\frac{1}{\hat{a}}+\frac{\beta_{2}}{\beta_{1}} \log (\hat{a})
$$

in order to match both sides of Eq. (9). The coupling $\hat{a}$ is then implicitly defined through

$$
\frac{1}{\hat{a}}+\frac{\beta_{2}}{\beta_{1}} \log (\hat{a})=\beta_{1} \log \left(\frac{Q}{\Lambda_{\mathrm{CCD}}}\right)+\frac{\beta_{1}}{2} C .
$$

The choice of $f$ is not arbitrary, but it is necessary so that the perturbative relation between $a$ and $\hat{a}$ remains a simple power expansion $\hat{a}=a+\sum_{n \geq 1} c_{n} a^{n+1}$. It is in this sense that $\hat{a}$ is a legitimate coupling redefinition. We call $\hat{a}$ the $C$-scheme coupling, which was first introduced in [7].

We note that in the large- $\beta_{0}$ approximation (where $\beta_{n}=0$ for all $n \geq 2$ ), $\hat{a}$ has the same scheme dependence as in Eq. (4).

\section{$C$-scheme coupling evolution}

The $C$-scheme coupling has simple properties regarding scale and $C$ transformations. Differentiating Eq. (11) with respect to either $C$ or $Q$, we find

$$
-Q \frac{\mathrm{d} \hat{a}}{\mathrm{~d} Q} \equiv \hat{\beta}(\hat{a})=\frac{\beta_{1} \hat{a}^{2}}{1-\frac{\beta_{2}}{\beta_{1}} \hat{a}}=-2 \frac{\mathrm{d} \hat{a}}{\mathrm{~d} C} .
$$

So changes in the scheme $C$ are completely equivalent to changes in the scale $Q$. A shift in the scale from $Q_{1}$ to $Q_{2}$ can be compensated by a shift in the scheme from $C_{1}$ to $C_{2}$ so that $Q_{1} / Q_{2}=e^{C_{1}-C_{2}}$.

In addition, because $\beta_{1}$ and $\beta_{2}$ are both scheme independent parameters, then the $\beta$ function of $\hat{a}$ is explicitly scheme independent.

\section{Cancellation of even $\zeta$ 's in perturbative expansions}

As an example for the discussion of this section, we consider the second derivative of the scalar correlator

$$
\Psi^{\prime \prime}\left(Q^{2}\right) \sim K \frac{m^{2}}{Q^{2}} \sum_{n \geq 0} b_{n} a^{n}
$$


with the scale choice $\mu^{2}=Q^{2}$ for both mass and coupling. The perturbative expansion in Eq. (13) is currently known up to fourth order. The coefficients $b_{n}$ contain different values of the Riemann $\zeta$ function $[8,9,10]$ :

$$
\begin{aligned}
& b_{2}=-\frac{35}{2} \zeta_{3}+\ldots \\
& b_{3}=\frac{715}{12} \zeta_{5}-\frac{5}{4} \zeta_{4}-\frac{65869}{216} \zeta_{3}+\ldots \\
& b_{4}=\frac{52255}{256} \zeta_{7}-\frac{625}{48} \zeta_{6}+\frac{59875}{108} \zeta_{5}-\frac{14575}{576} \zeta_{4}+\ldots
\end{aligned}
$$

We will rewrite the perturbative series of Eq. (13) in two steps. In the first step, we replace the mass $m$ by its scale-invariant version

$$
\widehat{m}=m(\pi a)^{-\gamma_{1} / \beta_{1}} \exp \left(\int_{0}^{a} \mathrm{~d} a\left[\frac{\gamma(a)}{\beta(a)}-\frac{\gamma_{1}}{\beta_{1} a}\right]\right)
$$

and obtain

$$
\Psi^{\prime \prime}\left(Q^{2}\right) \sim K \frac{\widehat{m}^{2}}{Q^{2}}(\pi a)^{2 \gamma_{1} / \beta_{1}} \sum_{n \geq 0} b_{n}^{\prime} a^{n},
$$

where the coefficients $b_{n}^{\prime}$ are combinations of the initial coefficients $b_{n}$ and contributions coming from the exponential factor in Eq. (17). The $\zeta_{4}$ present in $b_{3}$ and the $\zeta_{6}$ present in $b_{4}$ are cancelled by these contributions, but the $\zeta_{4}$ in $b_{4}$ still remains. The respective cancellations have also been observed in [11] for a related quantity.

In the second step, we replace in Eq. (18) the QCD coupling $a$ by the $C$-scheme coupling $\hat{a}$. The result is that the remaining $\zeta_{4}$ term in $b_{4}^{\prime}$ also cancels against a corresponding $\zeta_{4}$ present in the $\beta_{5}$ coefficient that arises from this replacement. Thus, the coefficients of the perturbative expansion become free of any even $\zeta$ term at least up to order $\hat{a}^{4}$ (although we expect this to be true to all orders in perturbation theory). This result has also been demonstrated for the gluonium correlator [12] and for several more physical quantities [13, 14].

\section{Borel models}

It is well known that perturbative expansions are divergent [15]. Adding more terms to an expansion would in general give better theoretical predictions, but there is a turning point when the factorial divergence of the coefficients dominate over the suppression of the coupling. From there, the precision degrades as more terms are added.

Conventionally, a finite value is assigned to the divergent expansions by considering its Borel sum:

$$
D(a)=\frac{2}{\beta_{1}} \int_{0}^{\infty} \mathrm{d} u e^{-2 u /\left(\beta_{1} a\right)} B[D](u)+\ldots
$$

However, the corresponding Borel transform $B[D]$ has singularities on the positive real axis which interfere with the Borel integral, producing imaginary ambiguities that are exponentially suppressed like $e^{-S / a}$, where $S>0$ is the position of the singularity. These ambiguities indicate the presence of opposing exponential corrections to the original perturbative expansion. We have

$$
D(a) \sim \sum_{n \geq 0} b_{n} a^{n} \pm i b e^{-S / a} a^{-\lambda} \sum_{n \geq 0} b_{n}^{\prime} a^{n}+\ldots, \quad a \rightarrow 0^{+},
$$


which is conventionally written as an operator product expansion (OPE) with the exponential factors written in terms of $\Lambda_{\mathrm{QCD}}^{2} / Q^{2}$. Each exponential factor corresponds to a different singularity $S$ in the Borel transform.

Imposing ambiguity cancellation between the two sectors of the expansion yields a relation between the large $n$ behaviour of the $b_{n}$ and the low $n$ behaviour of the $b_{n}^{\prime}$. This connection reads

$$
\begin{aligned}
b_{n} & =b \frac{(-1)^{n+1}}{\pi} \frac{\Gamma(n+\lambda)}{(-S)^{n+\lambda}}\left[1+\frac{-S b_{1}^{\prime}}{n+\lambda-1}+\mathscr{O}\left(\frac{1}{n^{2}}\right)\right], \\
& \pm i b e^{-S / a} a^{-\lambda}\left[a+b_{1}^{\prime} a^{2}+\mathscr{O}\left(a^{3}\right)\right] .
\end{aligned}
$$

Even if it is impossible to compute the coefficients $b_{n}$ at large $n$ from Feynman diagrams, we can still determine its large order behaviour thanks to this connection with the OPE. Namely, from the known structure of the OPE, we can fix the parameters $\lambda$ and $S$, and from the computation of the Wilson coefficients we can determine the $b_{n}^{\prime}$. But the residue $b$ can only be computed from the condensates and very little is known about them at the present time.

In [16], an ansatz is proposed for the Borel transform of the Adler function in full QCD. This ansatz incorporates the closest singularities to the origin $(u=-1,2,3)$ and their respective residues $b$ are fit with the first few known coefficients of the Adler function. This strategy already presumes that the large order behaviour of the $b_{n}$ sets in fast enough, but it is possible that a faraway singularity has an artificially high residue in such a way that its contribution to low orders is not negligible.

Using the $C$-scheme coupling, we want to improve on the above strategy by making sure that already at low order the large order behaviour is a good approximation to the true result. For that, we compute the Borel transform of the Adler function with respect to $\hat{a}$ (instead of $a$ ). The residues of this Borel transform change with the scheme $C$ and it is then possible to choose an optimal value of $C$ for which the residues of the closest singularities are enhanced with respect to far-away singularities.

The large- $\beta_{0}$ approximation is a good play field to qualitatively investigate how these changes take place. Going back to Eq. (2), we see that the residue at $u=S$ goes like $b \approx e^{-C S}$. Thus, as a rough approximation, $C>0$ enhances negative poles, while $C<0$ enhances positive poles. Lessons learned from this model then can be extrapolated to full QCD.

We hope theoretical uncertainties in $\alpha_{\mathrm{s}}$ extractions that arise from the truncation of perturbative expansions can be reduced by using the procedure described in this section.

\section{References}

[1] S. L. Adler, Phys. Rev. D 10 (1974) 3714.

[2] E. Braaten, S. Narison, and A. Pich, Nucl. Phys. B 373 (1992) 581.

[3] M. Beneke, Phys. Rept. 317 (1999) 1.

[4] M. Beneke, Nucl. Phys. B 405 (1993) 424.

[5] D. J. Broadhurst, Z. Physik C 58 (1993) 339.

[6] W. Celmaster and R. J. Gonsalves, Phys. Rev. D 20 (1979) 1420. 
[7] D. Boito, M. Jamin, and R. Miravitllas, Phys. Rev. Lett. 117 (2016) 152001.

[8] P. A. Baikov, K. G. Chetyrkin, and J. H. Kühn, Phys. Rev. Lett. 96 (2006) 012003.

[9] K. G. Chetyrkin, Phys. Lett. B 390 (1997) 309.

[10] S. G. Gorishnii, A. L. Kataev, S. A. Larin, and L. R. Surguladze, Mod. Phys. Lett. A 5 (1990) 2703.

[11] P. A. Baikov, K. G. Chetyrkin, and J. H. Kühn, JHEP 04 (2017) 119.

[12] M. Jamin and R. Miravitllas, Phys. Lett. B 779 (2018) 452.

[13] J. Davies and A. Vogt, Phys. Lett. B 776 (2018) 189.

[14] P. A. Baikov and K. G. Chetyrkin, JHEP 06 (2018) 141.

[15] F. J. Dyson, Phys. Rev. 85 (1952) 631.

[16] M. Beneke and M. Jamin, JHEP 09 (2008) 044. 